\title{
Meta-populational demes constitute a reservoir for large MHC allele diversity in wild house mice (Mus musculus)
}

\author{
Miriam Linnenbrink ${ }^{3 \dagger}$, Meike Teschke ${ }^{1,3+}$, Inka Montero ${ }^{2,3}$, Marie Vallier ${ }^{3}$ and Diethard Tautz ${ }^{3^{*}}$ (D)
}

\begin{abstract}
Background: The MHC class I and II loci mediate the adaptive immune response and belong to the most polymorphic loci in vertebrate genomes. In fact, the number of different alleles in a given species is often so large that it remains a challenge to provide an evolutionary model that can fully account for this.

Results: We provide here a general survey of MHC allele numbers in house mouse populations and two subspecies (M. m. domesticus and M. m. musculus) for $\mathrm{H} 2$ class I D and $\mathrm{K}$, as well as class II A and E loci. Between 50 and $90 \%$ of the detected different sequences constitute new alleles, confirming that the discovery of new alleles is indeed far from complete. House mice live in separate demes with small effective population sizes, factors that were proposed to reduce, rather than enhance the possibility for the maintenance of many different alleles. To specifically investigate the occurrence of alleles within demes, we focused on the class II H2-Aa and H2-Eb exon 2 alleles in nine demes of M. $m$. domesticus from two different geographic regions. We find on the one hand a group of alleles that occur in different sampling regions and three quarters of these are also found in both sub-species. On the other hand, the larger group of different alleles (56\%) occurs only in one of the regions and most of these (89\%) only in single demes. We show that most of these region-specific alleles have apparently arisen through recombination and/or partial gene conversion from already existing alleles.

Conclusions: Demes can act as sources of alleles that outnumber the set of alleles that are shared across the species range. These findings support the reservoir model proposed for human MHC diversity, which states that large pools of rare MHC allele variants are continuously generated by neutral mutational mechanisms. Given that these can become important in the defense against newly emerging pathogens, the reservoir model complements the selection based models for MHC diversity and explains why the exceptional diversity exists.
\end{abstract}

Keywords: Major histocompatibility complex, House mouse, Natural populations, Recombination, Reservoir model

\section{Background}

Genes encoded in the Major Histocompatibility Complex $(\mathrm{MHC})$ of vertebrates play key roles for the adaptive immune system. By presenting antigens of processed peptides from pathogens to $\mathrm{T}$ lymphocytes, proteins encoded in the MHC initiate a specific direct immune response. Among MHC Class I and II genes one can find the most polymorphic loci known in the vertebrate genome $[1,2]$. Despite decades of intense research, the evolutionary

\footnotetext{
* Correspondence: tautz@evolbio.mpg.de

${ }^{\dagger}$ Equal contributors

${ }^{3}$ Max-Planck Institute for Evolutionary Biology, August-Thienemannstrasse 2,

24306 Plön, Germany

Full list of author information is available at the end of the article
}

mechanisms of the origin and the maintenance of a large number of alleles in a given species are still much debated [3-11]. The two main adaptive factors that contribute to the maintenance of large $\mathrm{MHC}$ allele numbers are thought to be parasite-mediated selection and sexual selection [9], but theoretical studies have shown that these could account only for dozens to hundreds of alleles at a given locus $[6,7]$, but not the thousands that are nowadays found in deep surveys [12-14].

The most detailed data for MHC allele numbers are available for humans, since they are routinely analyzed in large cohorts to find matching donors for hematopoietic stem cell transplants. By surveying the human allele numbers, Klitz et al. [12] have suggested that the large numbers are 
due to recombination mechanisms that continuously generate new variants at low frequencies, but which are important to serve as a reservoir of alleles to fight new pathogens when they arise. The most recent re-analysis of human alleles has confirmed that millions of alleles exist in human populations, composed of a core set of ancient alleles, a much larger group of recombinant alleles (tens of thousands) and a long tail of extremely rare alleles that represent de novo point mutations in single individuals $[13,14]$. Robinson et al. [14] point out in their discussion that "... understanding their genetics and biology in any species requires extensive study of populations. For reasons of cost and logistics this has been rarely, if ever, achieved. Many population studies have recruited only small numbers of individuals (therefore, likely missing rare alleles)...". Our study here provides such an extended allelic survey in natural populations of the house mouse (Mus musculus), including a dedicated sampling scheme to explore MHC alleles within a metapopulational context.

The MHC complex in mice is located on chromosome 17 and is called $\mathrm{H} 2$ (or H-2) [15]. In a classic paper on heterozygosity of $\mathrm{H} 2$ loci in wild mice, Duncan et al. [16] found a close to $100 \%$ heterozygosity for class I loci and estimated the existence of at least 100 segregating alleles. However, this high heterozygosity stood in contrast to the known structure of inbreeding within demes. They wrote that "The intrademic inbreeding should lower the heterozygosity at the $\mathrm{H}-2$ loci..." and "The deme structure of the mouse population and the high degree of heterozygosity need to be reconciled" [16]. As a possible solution, they proposed that heterozygote advantage should offset the effect of inbreeding.

While a large number of segregating alleles in mice was later confirmed through various approaches [17-22] the analysis of allele distribution in demes was not revisited. Further, modeling of the heterozygote advantage has shown that it cannot explain the large number of alleles at $\mathrm{H} 2$ loci on its own [6, 7]. Rare-allele advantage and fluctuating selection may further contribute to the maintenance of larger allele numbers, but these factors are difficult to disentangle [9].

The phylogenetic and phylogeographic history of house mice has been well studied [23-25]. They have their origin in Iran or India and they have split into several subspecies within the past half million years. The focus in the present study are the Western (M. m. domesticus) and the Eastern (M. m. musculus) subspecies that have colonized their current territories only a few thousand years ago, as commensals with the spread of humans and establishment of agriculture. $M . m$. domesticus is thought to have arrived in Western Europe about 3000 years ago, originating from a distinct population in Western Iran [25-27]. The colonization of Eastern Europe and Northern Asia by M. $m$. musculus may have occurred a few thousand years earlier, possibly starting from a source population in Eastern Iran.
The M. m. domesticus spread across Western Europe has lead to a pattern of highly differentiated subpopulations [27-30]. In spite of their spatial differentiation, some gene flow occurs between populations and subspecies, apparently due to adaptive introgression of advantageous haplotypes across large distances [31, 32] as well as across the hybrid zone in Central Europe [33].

Here we use two sampling schemes to study MHC allele numbers in house mice. One is an overall survey across populations of the two sub-species, $M . m$. domesticus and M. m. musculus, the other is a specific survey of class II alleles within demes of two geographically distinct populations of $M$. $m$. domesticus. The overall survey yields many new alleles that were not previously described, but confirms also the sharing of a subset of alleles between the sub-species. Surprisingly, the survey of the demes revealed even higher allele numbers, with many alleles occurring only within single regions or demes and relatively little sharing between demes located next to each other. Analysis of these alleles suggest that they have arisen mostly through recombination mechanisms between existing alleles. Our data show that demes can serve as reservoirs of new alleles and support the inferences derived from human MHC allele surveys that at least thousands of rare alleles may exist that can become relevant for immune defense when new pathogens arise.

\section{Methods}

\section{Ethics and permissions}

This work did not involve research on animals that would require permission by an ethics committee. The trapping and keeping of animals was done under the permission of the authorities (permit from Veterinäramt Kreis Plön: 1401-144/PLÖ-004697), according to $\$ 11$ of the German animal welfare law (Tierschutzgesetz). Cervical dislocation was used when mice had to be sacrificed. Mice were either caught in snap traps, or live traps ("Mäusewippfalle" No. 3451002, Firma Ehlert \& Partner, 53,859 Niederkassel, Germany) and live mice were kept according to the FELASA guidelines as described in Harr et al. 2016 [27].

\section{Sampling}

Sample set 1 was used to generate a general overview of allele diversity, based on a 454 sequencing approach. We focused on two subspecies of the house mouse $(M . m$. domesticus and M. m. musculus (Fig. 1). M. m. domesticus is represented by individuals from Iran (IRA), from the Cologne/Bonn region in Germany (GER) and from the Massif Central region (FRA) in France. The population from Iran is considered to represent the ancestral source population for M. m. domesticus in Western Europe [25]. M. m. musculus is represented by individuals from the Czech Republic (CZE) and Kazakhstan (KAZ), whereby the KAZ population is considered to be the more ancestral one. DNA samples for sample set 1 were obtained from tissue of wild mice, caught in 


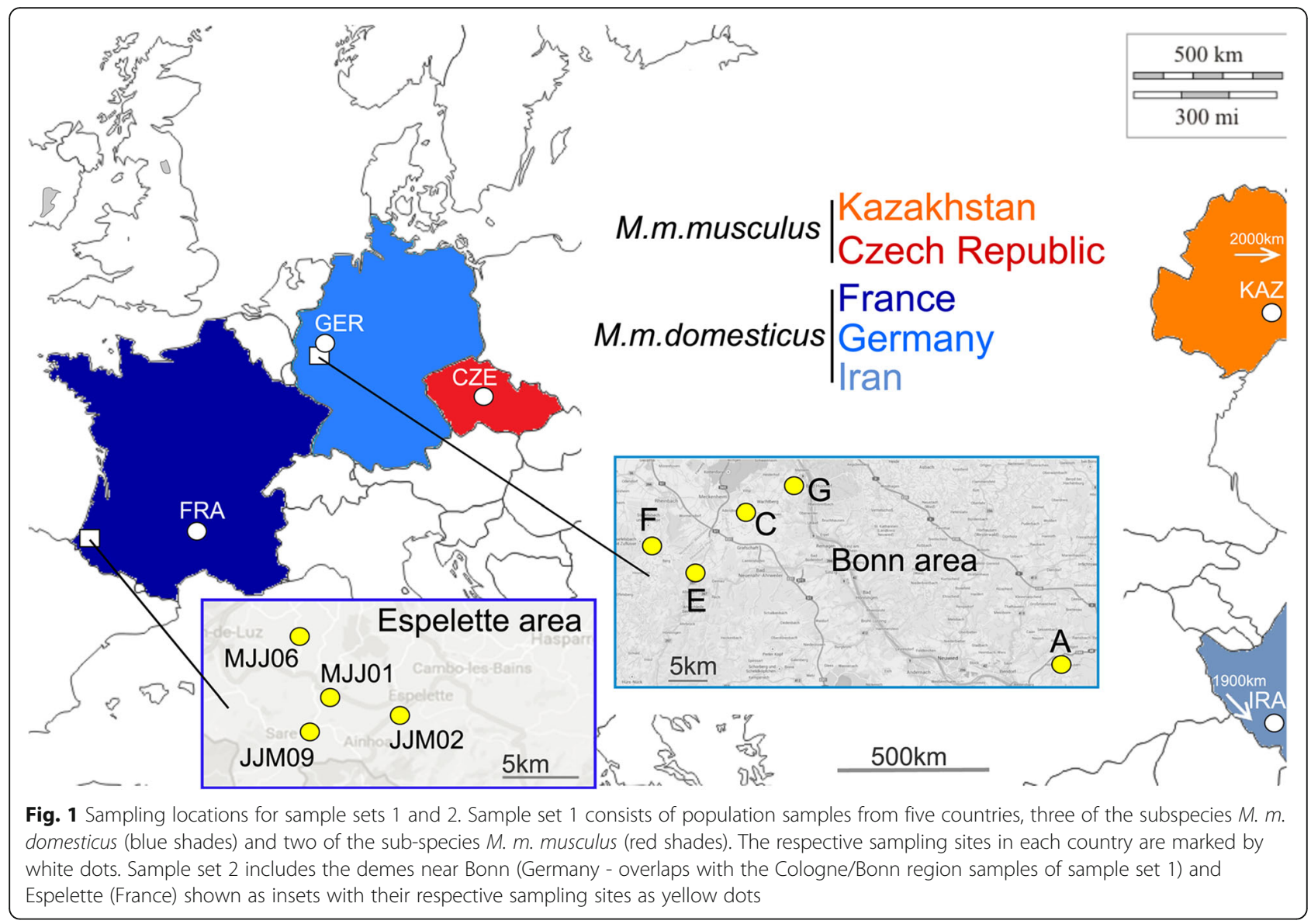

2004/2005 with a collection regime that aimed to sample the diversity across a region [28]. For the present study we used 12 individuals from GER, IRA, KAZ and CZE and 25 from the FRA population, in total 73 individuals.

Sample set 2 was used to assess the maintenance of allelic diversity in meta-populational demes. These samples were obtained from a region in Germany (Bonn) and a region in France (Espelette) (Fig. 1). The German region overlaps with the region of sample set 1, but the mice were caught in 2012. The French region was different from the one in sample set 1 and was sampled in 2013. Mice for sample set 2 were caught under a different sampling scheme than the mice for sample set 1 . Instead of maximizing diversity by using only a single mouse from each location, we aimed to obtain multiple individuals from the same locality, i.e., farms in sample set 2, including the possibility to catch some offspring from the same family. 6-18 individuals per farm were caught within a few days each, which resulted in a sample set of 47 mice from Bonn and 46 from Espelette. MHC alleles for these samples were determined by two different procedures, namely direct sequencing of PCR fragments for each individual and Illumina sequencing of PCR fragments from the same individuals.

\section{Targeted loci}

We focused on two MHC class I (H2-D and H2-K) and 4 MHC class II loci (H2-Aa, H2-Ab, H2-Ea and H2-Eb). We targeted the following exons: H2-D Exon 2 and 3, H2-K Exon 2, 3 and 4, H2-Aa Exon 2 and 3, H2-Ab Exon 2 and 3, $\mathrm{H} 2$-Ea Exon 2 and 3, H2- Eb Exon 2 and 3 (Additional file 1: File S1 provides an overview). Exons 2 and 3 of MHC class I loci and exon 2 of class II loci code for the antigen peptide binding groove. PCR primers were designed manually by searching exon flanking intronic regions for suitable sequences in the mouse reference sequence [34]. For each exon, up to four primer pair combinations were used for each animal that spanned the complete exons of each of the above mentioned loci to be able to confirm alleles in different PCR fragments. A list of all primers and PCR conditions used in this study is provided in Additional file 2: Table S1.

\section{Library preparation strategy}

$\mathrm{MHC}$ allele detection through parallel sequencing, such as 454 sequencing, is superior to more indirect methods such as techniques based on physical separation of alleles, e.g., single-stranded conformational polymorphism (SSCP), denaturing gel gradient electrophoresis (DGGE), or reference strand mediated conformational polymorphism (RSCA) 
[35]. But there are also drawbacks, such as errors introduced by the sequencing technique $[35,36]$, as well as PCR errors caused by the high levels of heterozygosity. PCR reactions to amplify MHC loci are effectively carried out with multi-allelic templates, which frequently cause problems, mainly through the formation of chimeras through incompletely extended primers, which act as primers in the subsequent PCR cycles $[37,38]$. Especially in the later cycles of the PCR, this chimera formation increases when the concentration of the incompletely extended primers is high enough to compete with the original primers for annealing. Thus, artificially recombined alleles are generated. This problem increases when templates of different individuals are amplified in the same PCR reaction, especially for the extremely polymorphic MHC Class II alleles. To minimize the problems caused by possible PCR artifacts, we reduced the number of PCR cycles to 28 and used independent amplifications. The first step of library preparation was the amplification of MHC exon fragments in individual PCRs. Each fragment was separately amplified from each individual in four independent reactions using the different primer combinations described above. After determining the concentrations of each PCR per locus via band intensity after gel electrophoresis and measuring the concentration on a Qubit fluorometer using the Quant-iT BR assay (Invitrogen), all fragments per individual were normalized to the same concentration and pooled. Pools were then tagged by ligating multiplex identification tags (MIDs) of $10 \mathrm{bp}$ to the fragments. The last step consisted of the ligation of amplicons with $\mathrm{Y}$ adapters and then 454 sequencing according to the standard protocol (Roche). A total of three pools were generated and each was run on one lane. Pool 1 encompassed 25 individuals of the FRA population and yielded 89,782 reads, pool 2 encompassed 12 individuals each of the CZE and KAZ populations and yielded 91,498 reads, pool 3 encompassed 12 individuals each of the GER and IRA populations and yielded 92,006 reads.

\section{Raw read processing}

After quality filtering of reads by applying the criteria of average quality score for bases $>25$ and read length $>$ $200 \mathrm{bp}$, without any "Ns", the remaining reads were edited with BioEdit. The MID-tags were cut off the sequences. Forward and reverse sequences were determined, primers and the intronic regions close to the exon were trimmed. Read statistics are provided in Additional file 3: Table S2. In the last step of data processing, all reads per locus were aligned to reference sequences obtained from database surveys using ClustalW [39]. Typical sequencing errors as described by Babik et al. [35] were manually edited in all full length reads (Additional file 1: File S1 for detailed description and examples). After raw read processing, alleles were identified as being reliable if the same sequences were found in at least two independent amplifications with different primers or in two different individuals. As a final step, the sequences were checked back against the un-edited raw reads. If at least two raw reads confirmed the sequence, it was accepted. All reliable alleles per locus are listed in Additional file 4: Table S3 (class I loci) and Additional file 5: Table S4 (class II loci).

\section{Sanger sequencing of exons}

The allele assignment for the sample set 2 individuals was done by direct sequencing of PCR fragments of H2-Aa and $\mathrm{H} 2$-Eb exon 2 from individuals through Sanger sequencing, which was previously shown to be efficient for determining the sequence of MHC alleles and which avoids also the problem of PCR recombination artifacts [40]. Primers and the PCR protocols are included in Additional file 2: Table S1. All individuals were sequenced separately and sequences were edited manually with Codon Code Aligner and aligned with ClustalW, included in the program MEGA 6 [41]. To provide raw sequence information for both loci two project files were generated in Codon Code Aligner. Heterozygous positions were identified based on double peaks in the sequence reads, both through automatic calling, as well as manual curation, since we noted that automatic calling does not detect all heterozygous positions. Haplotypic phase was determined using PHASE version 2.1 [40, 42, 43] and manually curated, based on known alleles. In some cases we used also the inequality of peak sizes in the overlapping sequences, due to unequal amplification of the alleles, as additional criterion for phasing.

\section{Illumina sequencing}

To further confirm the alleles and the correct phasing of alleles of set 2, we used an Illumina sequencing approach which can provide faithful results [44]. We amplified DNA from all sample set 2 individuals with the primers included in Additional file 2: Table S1, including unique barcoding tags. The fragments were then run on an Illumina MiSeq, using the long read kit to obtain single sequences across the whole region. Only individuals with $>5000$ reads were considered in the analysis. To detect whether one or two alleles were present, UPGMA trees of the aligned reads were constructed and checked whether one or two clusters were present. This allows to identify also cases where the two alleles were amplified very unequally. Consensus sequences were then generated across the reads of the clusters.

\section{Consolidation of sample set 2 reads}

The direct and Illumina obtained haplotypes for each individual were compared to see whether they validate each other. Conflicts were resolved by re-inspecting the original direct reads. However, we found also cases where the two approaches did not validate each other, apparently due to poor amplification of one of the two alleles. We had a few cases where the direct sequencing showed clear peaks (i.e. 
no indication of a second allele), while the Illumina reads showed clearly two alleles and vice versa. In some cases we could verify the second allele inferred from the direct sequencing by directly searching it among the Illumina reads, i.e. it was in these cases only very poorly amplified. We have therefore recorded for each individual whether the respective alleles were supported by both approaches, or only one of them (the latter also partly due to failure to get a result for the respective individual from one of the approaches) (Additional file 6: Table S5 and Additional file 7: Table: S6). Individuals for which we had no Illumina verification and for which we could extract no known allele from the direct sequencing were not considered in the final analysis, since phase could not be determined. This implies that we are in fact somewhat underestimating the number of alleles in the demes. Further, because of the different precautions and checks that were applied, we can practically rule out the possibility that some alleles may be due to PCR recombination artifacts.

All reliable alleles were compared with those identified in sample set 1 and with previously recorded alleles in databases using alignments and UPGMA trees generated in Geneious (Geneious.com). To make them comparable, alleles were all trimmed to the same length (208 bp for $\mathrm{H} 2-\mathrm{Aa}$ and $231 \mathrm{bp}$ for $\mathrm{H} 2-\mathrm{Eb})$, which is shorter that the whole exon due to missing data in some individuals.

\section{Microsatellite genotyping}

To infer relatedness among individuals of sample set 2 , we chose 10 unlinked microsatellite markers derived from Thomas et al. [45]. These markers are Chr3_24R, Chr16_ 21R, CHr12_05R, Chr01_25R, Chr17_09R, Chr05_45R, Chr13_22R, Chr19_08R, Chr14_16R, Chr09_20R. Primer information is given in Additional file 2: Table S1. Forward primers were labeled with FAM or HEX and PCR was performed using $5 \mathrm{ng} / \mu \mathrm{L}$ DNA template together with the Multiplex PCR kit (QIAGEN). After processing PCR products with $\mathrm{HiDi}$ formamide and 500 ROX size standard, samples were run on an ABI 3730 sequencer (Applied Biosystems). Raw alleles were called using GeneMapper 4. 0 (Applied Biosystems). The alleles are provided in Additional file 8: Table S7.

\section{Statistics}

Observed and expected heterozygosities for microsatellites were calculated using MSA version 4.05 [46] and for MHC alleles using GENODIVE [47]. Average relatedness was determined with the program COANCESTRY [48]. To investigate the structure between and within the farms we used the software STRUCTURE [49] for the microsatellite data. The parameters used were 500,000 burn-in period and 1,000,000 Markov Chain Monte Carlo (MCMC) simulations with 10 iterations per number of clusters (K) for K equals 215 . To determine the most likely number of populations that describe the existing amount of genetic structure, we used Structure Harvester [50] and applied the criterion of Evanno et al. [51]. Calculations of Hardy-Weinberg equilibrium tests between demes were performed in Arlequin [52]. Rarefaction analysis was done with the R-package "vegan" using the function "rarefy" with 10,000 bootstrap. dN/dS ratios were calculated in MEGA 6.0 [41], distances are based on the Kimura 2 Parameter model. The minimum number of recombination events and determination of sites between which recombination is inferred was investigated by using the software DNASp [53] and the genetic algorithm for recombination detection (GARD) as implemented on the webserver DATAMONKEY [54]. The software GENECONV (http:// www.math.wustl.edu/ sawyer) [55] was used to identify possible past gene conversion events. Significant gene conversion tracks were detected by permutation test $(1,000,000 \times)$ and $p$-values were corrected for multiple comparisons.

\section{D-loop sequencing and analysis}

A fragment of $\sim 980$ bp of the mitochondrial D-loop was sequenced. Primers used were taken from Prager et al. [56] (Additional file 2: Table S1). Sequences were edited manually with Seqman (DNASTAR, Inc., Madison, WI, USA). All sequences were aligned using the algorithm Clustal W and a Neighbor Joining Tree (bootstrap 1000×) was constructed using the program MEGA 6.0 [41].

\section{Data accessibility}

All data are provided in the supplementary files of this submission. New MHC allele sequences have been submitted to Genbank under accession numbers MF629153-MF629668 (see Additional file 9: Table S8 for the full list).

\section{Results}

\section{Population sampling}

We used two different sample sets: Sample set 1 represents a general survey, aimed to identify new MHC alleles in wild mice and involved the two subspecies $M . m$. domesticus and M. m. musculus, represented by sampling five different regions (depicted as white circles in Fig. 1). For this sample set we sequenced a number of exons from MHC class I (H2-D and H2-K) and MHC class II loci (H2-Aa, H2-Ab, H2-Ea and H2-Eb)(graphic overview and details are provided in Additional file 1: File S1). These sequences were then combined with sequences from the database and served as reference data set for the second part of the study.

Sample set 2 aimed to study the patterns of class II MHC allele distribution in a metapopulation context of $M . m$. domesticus, represented by local groups (demes) of mice from farms in two separate regions (depicted as yellow circles in the extended insets in Fig. 1). Note that the sampling strategy for these samples is different from that for sample set 1 . While sample set 1 aimed to obtain unrelated individuals 
across a region, i.e., only a single individual per deme was sampled, sample set 2 aimed to obtain multiple individuals from given demes within the respective regions. Further, for sample set 2 we restricted the sequence analysis to $\mathrm{H} 2-\mathrm{Aa}$ and $\mathrm{H} 2$-Eb exon 2 sequences, since the largest number of previously determined reference alleles from wild mice are available for these loci and since they allow to do direct sequencing of heterozygous individuals, due to the absence of indel polymorphisms.

\section{Survey of MHC alleles}

Based on the sequencing results across populations (sample set 1), we found for all sequenced loci $50-90 \%$ more alleles than previously recorded in the NCBI database (Table 1; full sequence lists in Additional file 4: Table S3 and Additional file 5: Table S4). Most alleles were found for class I loci (565 out of 684 for all exons). H2-D showed more alleles for exon 3 than exon 2, H2-K showed the opposite pattern. Both exons code for the domains forming the peptide binding groove. The largest number of alleles (197) were found for exon 4 of $\mathrm{H} 2-\mathrm{K}$, which codes for a part of the protein which is not directly involved in peptide binding. This exon is usually not surveyed in human studies [14], i.e. knowledge about its polymorphism in other systems is limited. About a third of these alleles appear to be non-functional due to frame shifts or premature stop codons. However, we noticed during the analysis that several individuals showed more than two alleles. We ascribe this to the possibility that the primers we used could also partially amplify paralogs of these genes (the annotated $\mathrm{Q}, \mathrm{T}$ and $\mathrm{M}$ loci - compare depiction in Additional file 1: File S1). These loci vary by

Table 1 Numbers of MHC H2 alleles found in the general survey (sample set 1)

\begin{tabular}{|c|c|c|c|c|c|c|}
\hline & \multirow[b]{2}{*}{ locus } & \multirow[b]{2}{*}{ exon } & \multirow[b]{2}{*}{ total } & \multicolumn{3}{|c|}{ fraction of: } \\
\hline & & & & $\overline{\text { new }^{a}}$ & silent $^{\mathrm{b}}$ & $\overline{\text { non functional }}$ \\
\hline \multirow[t]{5}{*}{ class I } & $\mathrm{H} 2-\mathrm{D}$ & 2 & 29 & 0.66 & 0.03 & 0 \\
\hline & & 3 & 120 & 0.90 & 0.10 & 0.23 \\
\hline & $\mathrm{H} 2-\mathrm{K}$ & 2 & 183 & 0.89 & 0.08 & 0.08 \\
\hline & & 3 & 36 & 0.64 & 0 & 0.11 \\
\hline & & 4 & 197 & 0.84 & 0.10 & 0.34 \\
\hline \multirow[t]{8}{*}{ class II } & $\mathrm{H} 2-\mathrm{Aa}$ & 2 & 20 & 0.50 & 0 & 0 \\
\hline & & 3 & 15 & 0.53 & 0.67 & 0 \\
\hline & $\mathrm{H} 2-\mathrm{Ab}$ & 2 & 41 & 0.88 & 0 & 0.10 \\
\hline & & 3 & 28 & 0.71 & 0.71 & 0.21 \\
\hline & $\mathrm{H} 2-\mathrm{Ea}$ & 2 & 7 & 0.57 & 0.29 & 0.14 \\
\hline & & 3 & 8 & 0.63 & 0 & 0.25 \\
\hline & $\mathrm{H} 2$-Eb & 2 & 55 & 0.73 & 0.04 & 0.13 \\
\hline & & 3 & 16 & 0.81 & 0.44 & 0.13 \\
\hline
\end{tabular}

${ }^{a}$ not previously recorded in the NCBI databank

${ }^{b}$ differing only in non-coding sequence positions from other alleles

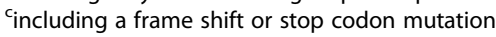

copy-number in natural populations [57] and it is therefore unpredictable which variants segregate in the populations. Hence, we can not be certain whether the H2-D and H2-K alleles which we identified here can indeed be ascribed to the respective loci or to their paralogs. On the other hand, since these paralogs appear to be similarly expressed (see expression data in [27]), they might be functionally equivalent. Still, because of the uncertainty of assignment, we did not analyze these alleles in further depth.

For the class II loci we had no assignment problem, as there are apparently no paralogs. There are none in the reference sequence and we never found more than two alleles per individual (note that this confirms further that PCR recombination artifacts can be largely excluded see also Methods). For these loci, we find a larger number of different alleles for exon 2 than for exon 3, with the exception of H2-Ea, which has generally fewer alleles than the three other loci (Table 1).

Since the class II alleles could be unequivocally assigned, we also analyzed their distribution patterns between the populations. Each population shows a subset of already described and new alleles and many are shared between at least one population of each of the subspecies (Table 2 and Additional file 5: Table S4). Interestingly, also alleles that differ only in non-coding positions and even nonfunctional alleles are partly shared between the subspecies (see annotations in Additional file 5: Table S4).

The largest number of different alleles were found in in the FRA population, which is represented by twice as many individuals as the other populations (Table 2), suggesting that more alleles are found with higher sampling depth, i.e. the discovery of new alleles is not exhausted.

\section{Deme structure of sample set 2 populations}

Sample set 2, collected around Bonn and Espelette (Fig. 1), was used to assess allele distributions among individuals

Table 2 Numbers of MHC H2 class II alleles in the different populations of sample set 1

\begin{tabular}{|c|c|c|c|c|c|c|c|c|}
\hline \multirow[b]{2}{*}{ Locus } & \multirow[b]{2}{*}{ exon } & \multirow[b]{2}{*}{ total } & \multicolumn{3}{|c|}{ M. m. domesticus } & \multicolumn{3}{|c|}{ M. m. musculus } \\
\hline & & & $\begin{array}{l}\operatorname{IRA}^{a} \\
N=12\end{array}$ & $\begin{array}{l}\mathrm{GER}^{a} \\
\mathrm{~N}=12\end{array}$ & $\begin{array}{l}\text { FRA }^{a} \\
N=25\end{array}$ & $\begin{array}{l}\mathrm{CZE}^{1} \\
\mathrm{~N}=12\end{array}$ & $\begin{array}{l}\mathrm{KAZ} Z^{\mathrm{a}} \\
\mathrm{N}=12\end{array}$ & shared $^{\mathrm{b}}$ \\
\hline \multirow[t]{2}{*}{$\mathrm{H} 2-\mathrm{Aa}$} & 2 & 20 & $4 / 0$ & $6 / 1$ & $14 / 2$ & $7 / 0$ & $6 / 0$ & 7 \\
\hline & 3 & 15 & $6 / 0$ & $7 / 1$ & $4 / 1$ & $8 / 1$ & $6 / 0$ & 7 \\
\hline \multirow[t]{2}{*}{$\mathrm{H} 2-\mathrm{Ab}$} & 2 & 41 & $11 / 6$ & $7 / 6$ & $16 / 7$ & $10 / 4$ & $11 / 5$ & 7 \\
\hline & 3 & 28 & $9 / 1$ & $11 / 2$ & $15 / 2$ & $19 / 2$ & $10 / 0$ & 17 \\
\hline \multirow[t]{2}{*}{$\mathrm{H} 2-\mathrm{Ea}$} & 2 & 7 & $4 / 0$ & $4 / 1$ & $5 / 0$ & $3 / 0$ & $3 / 0$ & 4 \\
\hline & 3 & 8 & $3 / 0$ & $3 / 1$ & $5 / 1$ & $3 / 0$ & $4 / 1$ & 4 \\
\hline \multirow[t]{2}{*}{$\mathrm{H} 2-\mathrm{Eb}$} & 2 & 55 & $16 / 5$ & $12 / 4$ & $30 / 9$ & $17 / 7$ & $14 / 2$ & 15 \\
\hline & 3 & 16 & $7 / 0$ & $7 / 0$ & $12 / 1$ & $6 / 2$ & $8 / 0$ & 9 \\
\hline
\end{tabular}

afirst number: total number of alleles found in the population / second number: number of population specific alleles

${ }^{b}$ number of alleles shared between at least one $M$. $m$. domesticus and at least one $M$. m. musculus population 
within and between demes. To confirm the deme structure of these locally sampled animals, we typed them for 10 microsatellites and determined mitochondrial D-loop sequences. We find that microsatellite heterozygosities are close to Hardy-Weinberg expectations, with deviations towards higher heterozygosity in two of the demes (Table 3), implying that there is no extreme inbreeding. Still, the average relatedness is relatively high in most demes, supporting the notion of a small effective population size in the demes, up to family level relationships. This leads to high genetic differentiation, even between closely spaced demes (average $\mathrm{F}_{\mathrm{ST}}=0.4$ for Bonn and 0.16 for Espelette - all pairwise $\mathrm{F}_{\mathrm{ST}}$ comparisons between demes in Additional file 10: Table S9).

These findings can be contrasted to the results when one samples only a single animal per deme across the region (same scheme as for sample set 1 ). In this case one finds a higher number of different microsatellite alleles for a region (e.g. about 10 instead of 2-3) and average observed heterozygosities are always lower than the expected heterozygosities (see Table 1 in [28]). Hence, the comparison between the two sampling schemes (within demes versus across demes) suggests differentiation of demes within a region, typical for metapopulations.

A distinct deme structure is also supported by the patterns found for the D-loop haplotypes, as well as STRUCTURE analysis based on the microsatellite data (Fig. 2). The overall results show that most animals within a deme share a particular D-loop haplotype, indicating a strong matrilineal structure (Fig. 2A). However, there is also some evidence for migration between the demes, such as single animals not fitting into their group or some D-loop haplotypes shared across groups. This is also evident from the STRUCTURE analysis. The pattern confirms a high genetic distinction of the demes, but also that at least a few animals were recently exchanged between some demes (Fig. 2B).

Hence, all data are compatible with a metapopulation structure with extended family groups living at each location and occasional exchange between demes.

\section{Allele patterns in sample set 2 demes}

As described above, for sample set 2, we restricted the analysis to class II exon 2 sequences for $\mathrm{H} 2-\mathrm{Aa}$ and $\mathrm{H} 2$ $\mathrm{Eb}$. This was done by direct sequencing of PCR fragments, even from heterozygous individuals. However, in cases of heterozygous individuals, it can be difficult to infer the phase of the respective alleles. Hence, we have applied several precautions to confirm the alleles, including resequencing by an Illumina-based approach (see Methods). For allele assignment, we compared the reliably inferred allele sequences with those from our survey, as well as the NCBI sequence database (including the sequences from [22]). This revealed many region-specific or even demespecific alleles that were not previously identified (Fig. 3, Additional file 6: Table S5 and Additional file 7: Table S6).

Different demes harbor between 0 to 7 region-specific alleles and 1 to 6 alleles shared between regions. These numbers do not depend much on sampling depth, since there is no correlation between the number of sampled individuals within a deme and the number of detected alleles, neither for the region-specific nor the shared alleles (overall $\left(R^{2}<0\right.$. 001) (Fig. 4a). This implies that within each deme we are approaching saturation of allele detection.

Most demes differ not significantly from HardyWeinberg equilibrium for the MHC alleles, and where they do, they show a lowered observed heterozygosity (Fig. 3).

For H2-Aa we find more different alleles in the Bonn region than in Espelette (22 versus 17), while the opposite trend is seen for $\mathrm{H} 2$-Eb alleles (13 versus 28 ), which constitutes a significant difference (two tailed Fisher's exact test: $p=0.04$ ). This confirms the observation of Cizkova et al. [22] that the two loci can evolve rather independently of each other. Note that this difference is mostly driven by the differences in region-specific alleles for these loci (Fig. 3).

There are also other notable differences between regionspecific and shared alleles. The majority of shared alleles (24 out of 27) were also found in dataset set 1 and 19 were found in at least one of the M. m. musculus populations

Table 3 Microsatellite population parameters

\begin{tabular}{|c|c|c|c|c|c|c|}
\hline region & deme & $\mathrm{N}$ & $\begin{array}{l}\text { observed } \\
\text { heterozygosity }\end{array}$ & $\begin{array}{l}\text { expected } \\
\text { heterozygosity }\end{array}$ & $\begin{array}{l}\text { effective number } \\
\text { of alleles }\end{array}$ & average relatedness \\
\hline \multirow[t]{5}{*}{ Bonn } & A & 7 & 0.51 & 0.52 & 2.2 & 0.38 \\
\hline & C & 9 & 0.50 & 0.50 & 2.2 & 0.47 \\
\hline & E & 10 & 0.46 & 0.45 & 1.9 & 0.5 \\
\hline & $\mathrm{F}$ & 18 & 0.62 & 0.55 & 2.3 & 0.37 \\
\hline & G & 13 & 0.60 & 0.56 & 2.5 & 0.35 \\
\hline \multirow[t]{4}{*}{ Espelette } & JJM02 & 10 & 0.71 & 0.74 & 3.7 & 0.16 \\
\hline & JJM09 & 11 & 0.86 & 0.78 & 4.2 & 0.15 \\
\hline & MJJ01 & 16 & $0.85^{*}$ & 0.72 & 3.6 & 0.34 \\
\hline & MJJ06 & 11 & $0.72^{* *}$ & 0.62 & 2.7 & 0.48 \\
\hline
\end{tabular}

significances: ${ }^{*}=p<0.05 ; * *=p<0.01$ 
a

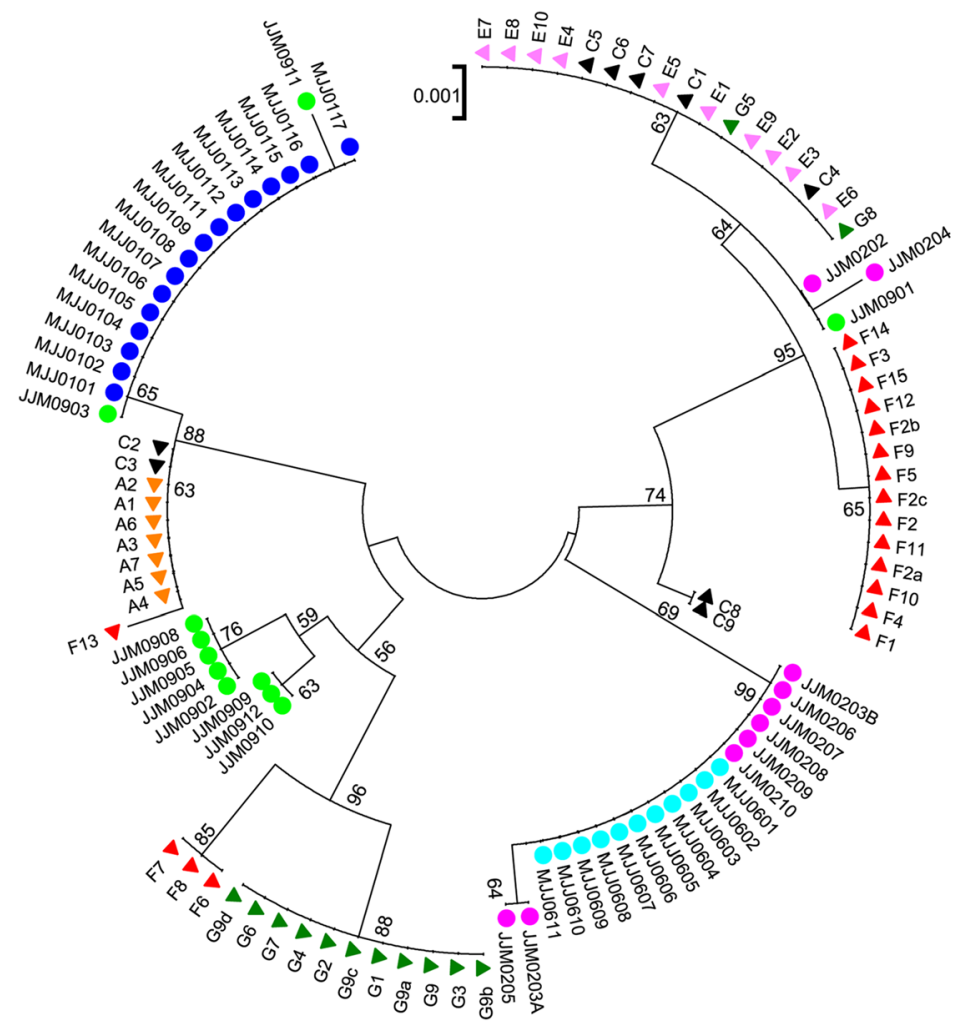

b

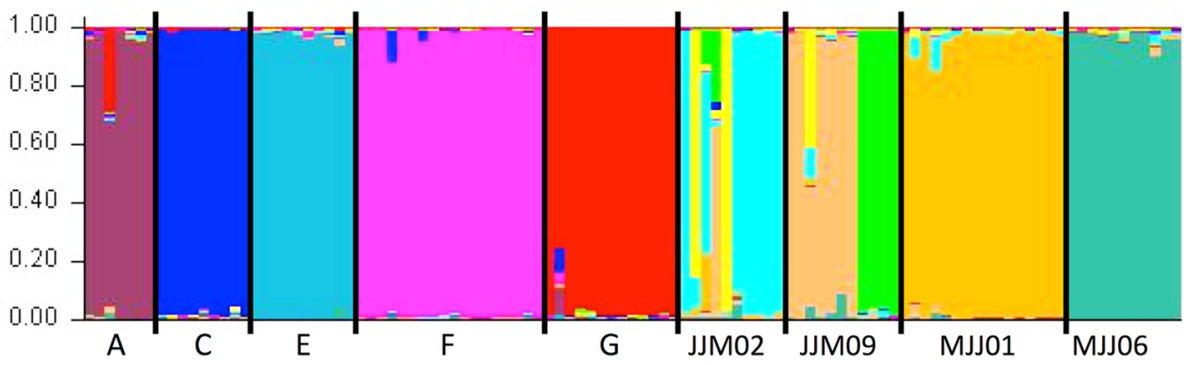

Fig. 2 Analysis of demes, based on D-loop sequences (a) and microsatellite analysis (b). The D-loop sequences show no sharing of haplotypes between France and Germany (circles vs. triangles), but much sharing of haplotypes within demes, confirming a strong matrilineal structure. On the other hand, several haplotypes are also shared between neighboring demes indicating some exchange in the past. The microsatellites were used to assess deme differentiation and degree of more recent exchange based on STRUCTURE analysis. The figure shows the results for $\mathrm{K}=11$ reflecting the optimal number out of 2-15. The demes JJM02 and JJM09 were split by STRUCTURE into two sub-demes, but with apparent exchange between them. Note that colors are only used for better visualization, they do not correspond between the figure parts

(see annotations in Fig. 3). In contrast, the majority (41 out of 46) of region-specific alleles were only found in a single deme in their respective region. This suggests that there may be two types of alleles, namely one group that occurs across populations in the whole species (including the different subspecies) and one group that is found more or less exclusively in local demes.

This could be a pure frequency effect, i.e. rare alleles are found by definition only in individual locations. Alternatively, it could correspond to the two classes of alleles that were postulated for humans, namely a core set of ancient alleles and a larger group of region-specific recombinant alleles [14]. If the latter is the case, one would predict that by sampling more demes, one should find increasing numbers of region-specific alleles, while the shared alleles should come to a saturation. Such a trend can indeed be traced in our data. Figure $4 \mathrm{~b}$ and c show rarefaction plots that indicate that sampling of shared alleles comes to a saturation for both loci, while sampling of region-specific alleles is still far from saturation.

\section{Sequence differences between alleles}

Most of the new alleles in the demes show amino acid differences compared to known alleles, only two new 


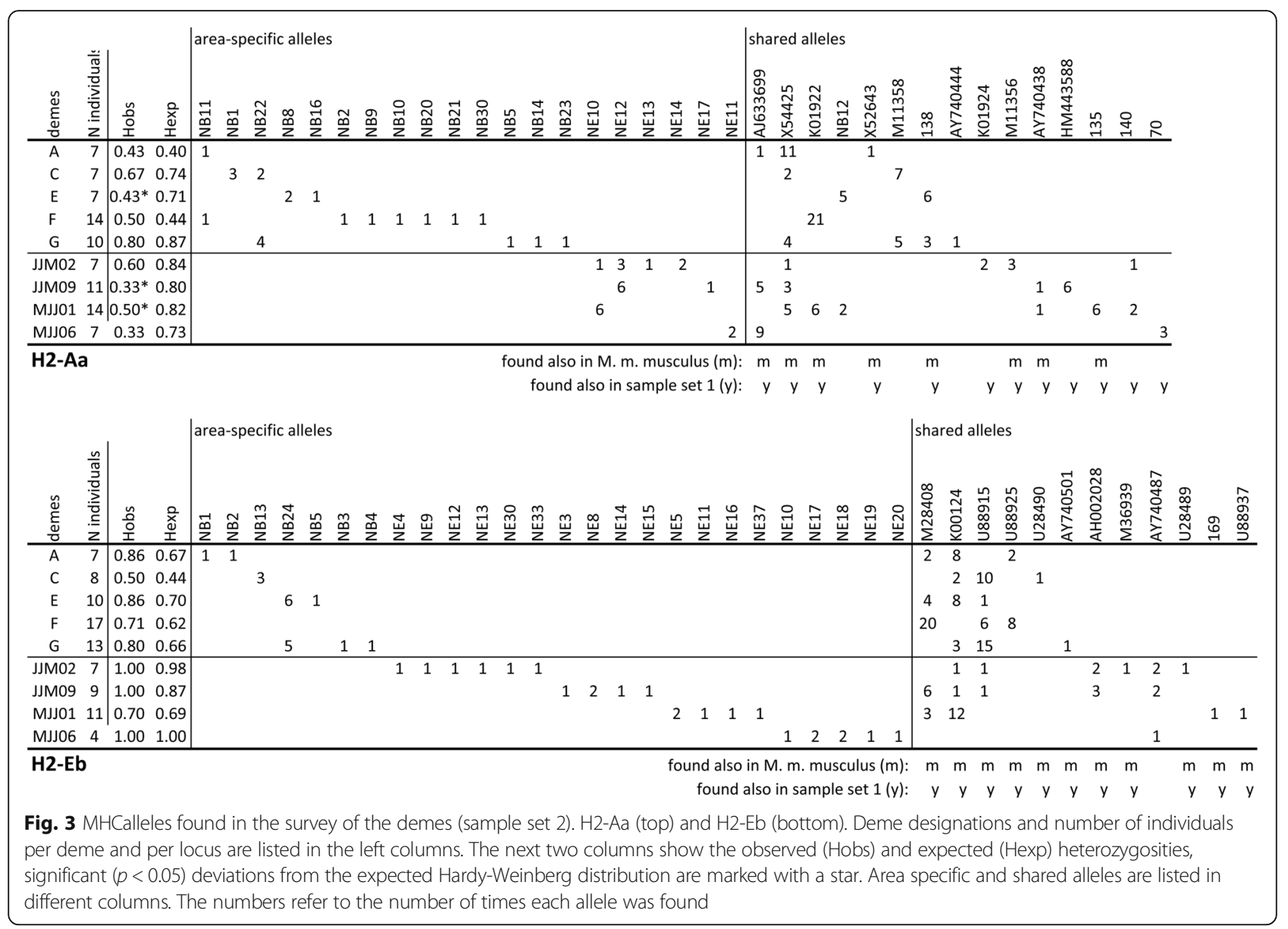

$\mathrm{H} 2$-Aa alleles and one new $\mathrm{H} 2$-Eb allele show noncoding differences. The amino acid differences are not randomly distributed across the sequenced region. Most occur at known hypervariable sites that constitute the contact sites of the peptide binding pocket of the antigen binding sites (ABS) (Additional file 11: Table S10 and Additional file 12: Table S11). Such biased substitution patterns are typical for MHC alleles and are ascribed to fast positive selection on sites that convey an advantage and negative selection or drift shaping the patterns of the other sites.

However, it is unlikely that such a mutation-selection process could have generated the new alleles within each of the single demes, since this would take an evolutionary time that would go beyond the life-time of these demes. Even when assuming a point mutation rate of $2 \times 10^{-8}$ and a population size of up to 1000 per deme, one can expect a new mutation to occur within the about $200 \mathrm{bp}$ region surveyed only every 2500 generations. Given that the life time of such a deme may be no more than 1000 years, there would simply not be enough new mutations to reach a mutation/selection equilibrium. Hence, recombination or partial gene conversion between existing alleles, either presently or previously present in the respective deme, is the much more likely explanation for the generation of the region-specific alleles.

We used several analysis algorithms to assess such possible recombination patterns. Given the likely fast allele turnover in these small demes (i.e. loss through drift) and the occasional introgression from neighboring demes (see above), one would not expect that the actual donor sequences for a given recombination event would be found in all cases. Hence, we included all known alleles (obtained from the NCBI database and sample set 1) as possible donors in the analysis of the alleles in each deme. Multiple recombination events could be detected by the four gamete test implemented in DNASp for both loci and most demes (Table 4). Significant sites of recombination in individual demes are detected by GARD analysis (Table 4).

Using the program GENECONV, we found also support for a number of partial gene conversion events within the demes (Table 5). Three significant events were found for $\mathrm{H} 2-\mathrm{Aa}$ and one for $\mathrm{H} 2-\mathrm{Eb}$, but only in the Espelette region.

\section{Discussion}

Our study confirms the original findings of Duncan et al. [16], namely a high number of MHC alleles in wild caught 


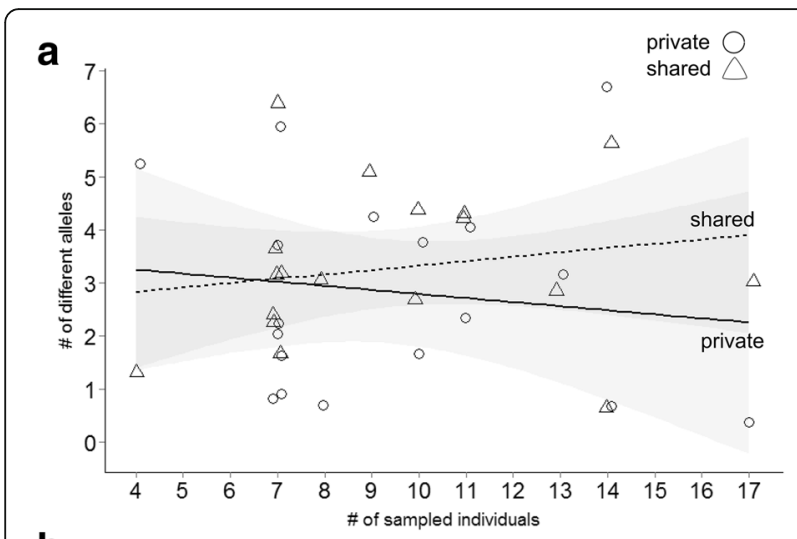

b

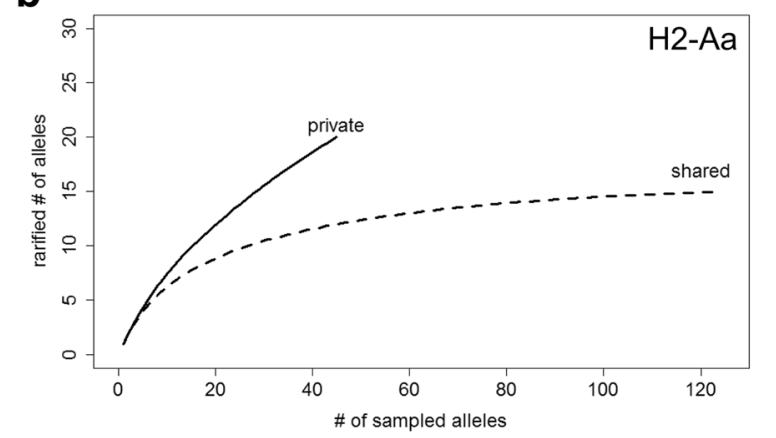

C

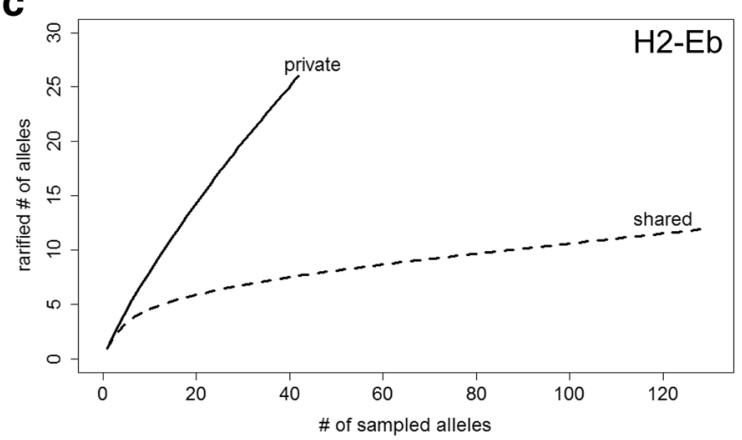

Fig. 4 Sampling statistics. (a) Correlation between number of sampled individuals per deme and number of alleles found for shared and region-specific alleles. The correlation lines are non-significant (grey area represents the 95\% confidence interval). (b) and (c) Rarefaction curves for the probability of discovery of new alleles with successive sampling. Only sampling of shared alleles comes to a saturation in our data. Note that shared alleles have a higher frequency, i.e. the total numbers are larger than for region-specific alleles, although the number of different alleles is smaller

mice, and that class I alleles are more polymorphic than class II alleles. However, class I allelic diversity is also influenced by paralogs with variable copy-numbers, which were not known at that time. Hence, the serological analysis done at that time could have picked up variants from expressed paralogs, i.e. the true degree of heterozygosity at these loci remains unclear. Given these complications, we have not explored them further in this study. Duncan et al. [16] also found a difference in allelic diversity between class II A and E loci, with relatively fewer alleles for locus E. We can confirm this for the H2-Ea locus, while we find that $\mathrm{H} 2-\mathrm{Eb}$ is on average highly polymorphic in our survey (compare Table 2).

We find many alleles that are shared across subspecies, which is a pattern generally known for MHC alleles $[3,18]$. These have usually been ascribed to be the result of balancing selection and/or incomplete lineage sorting after the splitting of the species. However, for species that have remained at least partially inter-fertile, there is increasing evidence that introgression must play a role as well [58-61]. In fact, geographical long-range transfer of haplotypes and adaptive introgression across sub-species was also found for other parts of the genome in mice [31]. In humans it is also thought that some alleles of the immune system are derived from introgression events among archaic lineages [58].

The mitochondrial and microsatellite analysis of the deme structure confirms the notion that these are composed of extended family groups, with average relatedness values up to 0.5 , but still largely in Hardy-Weinberg equilibrium. Intriguingly, and contrary to the expectations by Duncan et al. [16] that this deme structure should reduce allelic MHC diversity due to inbreeding and drift, we actually find more different region-specific alleles than shared alleles among these demes, although most of them are at a low frequency. Previous studies may have largely missed these low frequency alleles, because of less deep sampling, or because of the use of filtering steps that would have removed them as possible PCR artifacts. We applied such filters also for the sample set 1 study. In particular, we required at least two independent PCRs with the same sequence from at least two animals, which biases against rare alleles, since more abundant alleles are more likely to be found repeatedly. No such filter was used for sample set 2 (i.e. the alleles in the demes), since these were detected through direct sequencing and confirmed by Illumina sequencing of individuals, which allows the reliable identification of alleles that occur only in a single individual.

There is little sharing of region-specific alleles (5 out of 46) even between neighboring demes. In contrast, the shared alleles are not only shared more often within each region (16 out of 27), but most of them are also found in the populations of set 1 (23 out of 27) and across subspecies (19 out of 27). This is not simply an effect of relative frequencies. Although region-specific alleles tend to have a low frequency in demes, their probability of discovery does not correlate with sample size in the demes (Fig. 4a), i.e. we expect that the sampling is reasonably representative for each deme. Further, the rarefaction analysis suggests that sampling saturation is not reached for region-specific alleles (Fig. 4b, c), i.e. they can be predicted to occur in increasingly large numbers when more demes are sampled. Hence, considering the multitude of different demes in which mice 
Table 4 Estimated recombination events among alleles for each deme

\begin{tabular}{|c|c|c|c|c|c|}
\hline Location & Deme & $\begin{array}{l}\text { minimum number of } \\
\text { recombination events }\end{array}$ & $\begin{array}{l}\text { significant positions } \\
\text { found by GARD }\end{array}$ & $\begin{array}{l}\text { minimum number of } \\
\text { recombination events }\end{array}$ & $\begin{array}{l}\text { significant positions }^{\mathrm{b}} \\
\text { found by GARD }\end{array}$ \\
\hline & & \multicolumn{2}{|c|}{$\mathrm{H} 2-\mathrm{Aa}$} & \multicolumn{2}{|c|}{$\mathrm{H} 2-\mathrm{Eb}$} \\
\hline \multirow[t]{5}{*}{ Bonn } & A & 0 & - & 1 & - \\
\hline & C & 2 & & 3 & \\
\hline & E & 0 & - & 3 & \\
\hline & $\mathrm{F}$ & 3 & - & 0 & \\
\hline & G & 3 & - & 1 & \\
\hline \multirow[t]{6}{*}{ Espelette } & JJM02 & 8 & $156^{* *}$ & 9 & $50^{* *}$ \\
\hline & JJM09 & 2 & $156^{* * *}$ & 4 & $187^{* * *}$ \\
\hline & MJJ01 & 3 & $62^{*}$ & 4 & $181^{* * *}$ \\
\hline & MJJ06 & 0 & - & 9 & $188^{* * *}$ \\
\hline & all demes & 10 & - & 10 & - \\
\hline & all demes + all known alleles & 12 & - & 13 & - \\
\hline
\end{tabular}

aposition numbering refers to the sequences in Additional file 7: Table S6 a position numbering refers to the sequences in Additional file 8: Table S7 ${ }^{*} p<0.05 ;{ }^{* *} p<0.01 ;{ }^{* * *} p<0.001$

can be found across their whole distribution range, one may conclude that region-specific alleles should by far outnumber shared alleles.

This raises the question how the region-specific alleles arise in the demes. The analysis of their sequence differences suggests that they are mostly not created by new point mutations, but by recombination and/or partial gene conversion from existing alleles. These mechanisms were first described in detail for human class I loci [3], whereby the donors for the recombination or partial gene conversion were suggested to come mostly from intragenomic paralogs. In the mouse, there are no paralogs for the class II H2-Aa and $\mathrm{H} 2-\mathrm{Eb}$ loci that could serve as donors. Although a duplicate exists for $\mathrm{H} 2-\mathrm{Eb}$ (annotated as Eb1 and Eb2), this is molecularly too diverged to allow recombination to happen. Hence, the donors for recombination would have to be other alleles segregating within the demes, or coming in via occasional migration. We find indeed evidence for such recombination and partial gene conversion events within demes when taking all known alleles of the orthologs into account (see Tables 4 and 5). A further possibility are short tracks of gene conversion even between distant paralogs. The $A \beta^{\text {bm12 }}$ allele in the C57BL/6 laboratory strain has been suggested to have been created by a micro gene conversion event between $\mathrm{H} 2-\mathrm{A}$ and $\mathrm{H} 2-\mathrm{E}$ [62], i.e. this appears to work even between very diverged copies.

Recombination and partial gene conversion among MHC alleles has been documented in many other cases (e.g. [14, 22, 63-69]. Spurgin et al. [69] showed rather directly that allelic diversity is re-created through partial gene conversion events after bottlenecks in birds. Bergström et al. [66] and Spurgin et al. [69] estimated that the generation of new alleles through recombination mechanisms is at least one order of magnitude faster than through point mutations. Direct measurements of gene conversion rates between HLA genes in sperm have yielded an estimate two orders of magnitude higher than the point mutation rate in mice [64] and more than three orders of magnitude higher in humans [65]. This fits well with our observation that point mutations are rare in comparison to recombination patterns.

Exceptionally deep sampling of MHC diversity has been done in humans, since this is essential for matching donors and recipients for hematopoietic stem cell transplantations. Klitz et al. [12] have proposed that this diversity is mostly shaped by recombination mechanisms that create continuously rare variants and generate in this way a reservoir of new alleles that are likely to be

Table 5 Partial gene conversion events among alleles within demes

\begin{tabular}{lllllllll}
\hline locus & deme & kind $^{\text {a }}$ & allele 1 & allele 2 & simulated $p$-value & Begin & End & Length \\
\hline H2-Aa & JJM02 & Gl & NE12 & M11356 & 0.0016 & 57 & 183 & 127 \\
& JJM02 & Gl & NE13 & M11356 & 0.0431 & 112 & 183 & 72 \\
& JJM09 & Gl & NE12 & AY740438 & 0.0442 & 28 & 183 \\
H2-Eb & MJJ01 & GO & NE16 & & 0.0422 & 28 & 42 & 156 \\
\hline
\end{tabular}

${ }^{\mathrm{a}} \mathrm{Gl}$ : global inner fragment $=$ runs of matching sites; GO: global outer fragment $=$ runs of matching sites unique in that group 
pre-adapted for presentation of new pathogen-derived peptides. They provide some basic population genetic calculations implying that the human population as a whole might harbor millions of alleles at present. But this calculation depends on many assumptions about effective population size, equilibrium state, mutation rate, deme sizes and migration rates. Hence, while the same parameters might apply to mice, we refrain from such calculations since they are too speculative. However, it would not seem unlikely that mice as a whole might also harbor similar numbers of MHC alleles as humans.

A recent detailed analysis of human class I alleles in databases has also suggested that millions of alleles appear to exist [14]. This was estimated on the basis of the discovery rate of new alleles in tested cohorts, which was found to lie around 2 per 10,000 individuals tested. This is relatively low compared to our discovery rate of around 2.7 new alleles per 10 individuals (across all demes and loci), i.e. the mouse sampling must indeed be far from saturation.

In humans there is also evidence for a set of common alleles occurring at elevated frequencies and across populations [70]. This is comparable to the set of shared alleles that we find in mice, most of which occur also across subspecies. This suggests that they have a specific adaptive value, which maintains them or allows them to introgress between the diverse lineages. On the other hand, the rare alleles occur only locally, both in humans $[14,70]$ and mice (this study).

\section{Conclusion}

Although recombination mechanisms have long been known to contribute to allelic diversity at MHC loci, the current results suggest that it generates a large number of new alleles in demes, with much higher efficiency than point mutations would do this. However, most may also get quickly lost, due to small effective population sizes in the demes. Still, they constitute a reservoir of possible resistance alleles when new parasites come along. This leads to a new paradigm on how we should view the generation and maintenance of the diversity at MHC loci [12]. Given that these patterns match between mice and humans $[12,14]$, it would seem likely that they are generally typical for animals harboring the MHC adaptive immune system.

\section{Additional files}

Additional file 1: File S1. Experimental approach for sample set 1 survey (PPT $3321 \mathrm{~kb}$ )

Additional file 2: Table S1. PCR and sequencing primers (XLSX $17 \mathrm{~kb}$ )

Additional file 3: Table S2. 454 read statistics (XLSX 38 kb)

Additional file 4: Table S3. List of all validated class I alleles found in the 454 approach (XLSX $80 \mathrm{~kb}$ )
Additional file 5: Table S4. List of all validated class II alleles found in the 454 approach (XLSX 58 kb)

Additional file 6: Table S5. H2-Aa alleles in demes (XLSX 24 kb) Additional file 7: Table S6. H2-Eb alleles in demes (XLSX $24 \mathrm{~kb}$ ) Additional file 8: Table S7. Microsatellite alleles in demes (XLSX 20 kb) Additional file 9: Table S8. Genbank accession numbers for new alleles (XLSX $46 \mathrm{~kb}$ )

Additional file 10: Table S9. Pairwise distances between demes based on microsatellites (FST) (XLSX $37 \mathrm{~kb}$ )

Additional file 11: Table S10. Alignments of $\mathrm{H} 2-\mathrm{Aa}$ and $\mathrm{H} 2-\mathrm{Eb}$ alleles (XLSX $47 \mathrm{~kb}$ )

Additional file 12: Table S11. $d N / d S$ and K2P nucleotide distance/ Poisson corrected amino acid distances (XLSX 52 kb)

\section{Acknowledgments}

Many thanks go to Tobias Lenz, Jamie Winternitz, Primrose Boynton, Jun Wang and Anja Schunke for helpful comments. Thanks also to Heinke Buhtz for her technical assistance.

Authors' contributions

MT an IM devised the experiment and generated the sequences for sample set 1, MT and MV sampled the animals for smaple set 2, ML and MV generated the sequences and microsatellite data for sample set $2, M T, M L$ and DT did the analysis, ML and DT wrote the manuscript. All authors read and approved the final manuscript.

\section{Competing interests}

The authors declare that they have no competing interests.

\section{Publisher's Note}

Springer Nature remains neutral with regard to jurisdictional claims in published maps and institutional affiliations.

\section{Author details}

${ }^{1}$ Present address: Deutsche Forschungsgemeinschaft, 53170 Bonn, Germany. ${ }^{2}$ Present address: Medical Faculty, Eberhard Karls Universität Tübingen, Tübingen, Germany. ${ }^{3}$ Max-Planck Institute for Evolutionary Biology, August-Thienemannstrasse 2, 24306 Plön, Germany.

Received: 1 March 2018 Accepted: 10 April 2018

Published online: 20 April 2018

\section{References}

1. Klein J, Sato A, Nikolaidis N. MHC, TSP, and the origin of species: From immunogenetics to evolutionary genetics. Annu Rev Genet. 2007;41:281304. https://doi.org/10.1146/annurev.genet.41.110306.130137. PubMed PMID: WOS:000252359500013.

2. Robinson J, Halliwell JA, Hayhurst JD, Flicek P, Parham P, Marsh SGE. The IPD and IMGT/HLA database: allele variant databases. Nucleic Acids Res. 2015;43(D1):D423-DD31. https://doi.org/10.1093/nar/gku1161. PubMed PMID: WOS:000350210400065.

3. Parham P, Ohta T. Population biology of antigen presentation by MHC class I molecules. Science. 1996;272(5258):67-74. https://doi.org/10.1126/science. 272.5258.67. PubMed PMID: WOS:A1996UD59700038.

4. Apanius $V$, Penn D, Slev PR, Ruff $L R$, Potts WK. The nature of selection on the major histocompatibility complex. Crit Rev Immunol. 1997;17(2):179224. PubMed PMID: WOS:A1997WR04600004.

5. Takahata N, Satta Y. Improbable truth in human MHC diversity? Nat Genet. 1998;18(3):204-6. https://doi.org/10.1038/ng0398-204. PubMed PMID: WOS: 000072325000005.

6. De Boer RJ, Borghans JAM, van Boven M, Kesmir C, Weissing FJ. Heterozygote advantage fails to explain the high degree of polymorphism of the MHC. Immunogenetics. 2004;55(11):725-31. https://doi.org/10.1007/ s00251-003-0629-y. PubMed PMID: WOS:000189202400001.

7. Borghans JAM, Beltman JB, De Boer RJ. MHC polymorphism under hostpathogen coevolution. Immunogenetics. 2004;55(11):732-9. https://doi.org/ 10.1007/s00251-003-0630-5. PubMed PMID: WOS:000189202400002. 
8. van Oosterhout C. A new theory of MHC evolution: beyond selection on the immune genes. Proc R Soc B Biol Sci. 2009;276(1657):657-65. https://doi. org/10.1098/rspb.2008.1299. PubMed PMID: WOS:000262419000008.

9. Spurgin LG, Richardson DS. How pathogens drive genetic diversity: MHC, mechanisms and misunderstandings. Proc R Soc B Biol Sci. 2010;277(1684): 979-88. https://doi.org/10.1098/rspb.2009.2084. PubMed PMID: WOS: 000274858500001

10. Winternitz JC, Minchey SG, Garamszegi LZ, Huang S, Stephens PR, Altizer S. Sexual selection explains more functional variation in the mammalian major histocompatibility complex than parasitism. Proc R Soc B Biol Sci. 2013; 280(1769). https://doi.org/10.1098/rspb.2013.1605. PubMed PMID: WOS: 000330322000013

11. Kamiya T, O'Dwyer K, Westerdahl H, Senior A, Nakagawa S. A quantitative review of MHC-based mating preference: the role of diversity and dissimilarity. Mol Ecol. 2014;23(21):5151-63. https://doi.org/10.1111/mec. 12934. PubMed PMID: WOS:000344374000004.

12. Klitz W, Hedrick P, Louis EJ. New reservoirs of HLA alleles: pools of rare variants enhance immune defense. Trends Genet. 2012;28(10):480-6. https:// doi.org/10.1016/j.tig.2012.06.007. PubMed PMID: WOS:000309505200003.

13. Slater N, Louzoun Y, Gragert L, Maiers M, Chatterjee A, Albrecht M. Power Laws for Heavy-Tailed Distributions: Modeling Allele and Haplotype Diversity for the National Marrow Donor Program. Plos Comput Biol. 2015;11(4). https://doi.org/10.1371/journal.pcbi.1004204. PubMed PMID: WOS: 000354517600047

14. Robinson J, Guethlein LA, Cereb N, Yang SY, Norman PJ, Marsh SGE, et al. Distinguishing functional polymorphism from random variation in the sequences of > 10,000 HLA-A, -B and -C alleles. Plos Genet. 2017;13(6). https://doi.org/10. 1371/journal.pgen.1006862. PubMed PMID: WOS:000404512600039.

15. Kumanovics A, Takada T, Lindahl KF. Genomic organization of the mammalian Mhc. Annu Rev Immunol. 2003;21:629-57. https://doi.org/10. 1146/annurev.immunol.21.090501.080116. PubMed PMID: WOS: 000182523500019

16. Duncan WR, Wakeland EK, Klein J. HETEROZYGOSITY OF H-2 LOCI IN WILD MICE. Nature. 1979;281(5732):603-5. https://doi.org/10.1038/281603a0. PubMed PMID: WOS:A1979HQ78400071.

17. Gotze D, Nadeau J, Wakeland EK, Berry RJ, Bonhomme F, Egorov IK, et al. histocompatibility-2 system in wild mice .10. Frequencies of h-2 and ia antigens in wild mice from europe and africa. J Immunol. 1980;124(6):267581. PubMed PMID: WOS:A1980JT75200025.

18. Arden B, Klein J. Biochemical-comparison of major histocompatibility complex-molecules from different subspecies of mus-musculus - evidence for trans-specific evolution of alleles. Proc Natl Acad Sci U S A Biol Sci. 1982; 79(7):2342-6. https://doi.org/10.1073/pnas.79.7.2342. PubMed PMID: WOS: A1982NJ77700043

19. Nadeau JH, Brittondavidian J, Bonhomme F, Thaler L. h-2 polymorphisms are more uniformly distributed than allozyme polymorphisms in naturalpopulations of house mice. Genetics. 1988;118(1):131-40. PubMed PMID: WOS:A1988L692100014.

20. Edwards SV, Chesnut K, Satta Y, Wakeland EK. Ancestral polymorphism of Mhc class II genes in mice: Implications for balancing selection and the mammalian molecular clock. Genetics. 1997;146(2):655-68. PubMed PMID: WOS:A1997XD21600020.

21. Mitchison NA, Mayer W. A survey of $\mathrm{H} 2$ gene sequences, including new wild-derived genes. Int J Immunogenet. 2007;34(1):3-12. https://doi.org/10 1111/j.1744-313X.2006.00640.x. PubMed PMID: WOS:000244056100002.

22. Cizkova D, de Bellocq JG, Baird SJE, Pialek J, Bryja J. Genetic structure and contrasting selection pattern at two major histocompatibility complex genes in wild house mouse populations. Heredity. 2011;106(5):727-40. https:/doi. org/10.1038/hdy.2010.112. PubMed PMID: WOS:000289713700005.

23. Guenet $\mathrm{L}$, Bonhomme F. Wild mice: an ever-increasing contribution to a popular mammalian model. Trends Genet. 2003;19(1):24-31. https://doi.org/10.1016/ s0168-9525(02)00007-0. PubMed PMID: WOS:000180294900006.

24. Suzuki H, Nunome M, Kinoshita G, Aplin KP, Vogel P, Kryukov AP, et al. Evolutionary and dispersal history of Eurasian house mice Mus musculus clarified by more extensive geographic sampling of mitochondrial DNA. Heredity. 2013;111(5):375-90. https://doi.org/10.1038/hdy.2013.60. PubMed PMID: WOS:000325868000003.

25. Hardouin EA, Orth A, Teschke M, Darvish J, Tautz D, Bonhomme F. Eurasian house mouse (Mus musculus L.) differentiation at microsatellite loci identifies the Iranian plateau as a phylogeographic hotspot. BMC Evol Biol. 2015;15. https:// doi.org/10.1186/s12862-015-0306-4. PubMed PMID: WOS:000350063800001.
26. Cucchi T, Vigne JD, Auffray JC. First occurrence of the house mouse (Mus musculus domesticus Schwarz \& Schwarz, 1943) in the Western Mediterranean: a zooarchaeological revision of subfossil occurrences. Biol J Linn Soc. 2005; 84(3):429-45. https://doi.org/10.1111/j.1095-8312.2005.00445.x. PubMed PMID: WOS:000228097600011.

27. Harr B, Karakoc E, Neme R, Teschke M, Pfeifle C, Pezer Z, et al. Genomic resources for wild populations of the house mouse, Mus musculus and its close relative Mus spretus. Scientific Data. 2016;3. https://doi.org/10.1038/ sdata.2016.75. PubMed PMID: WOS:000390231600001.

28. Ihle S, Ravaoarimanana I, Thomas M, Tautz D. An analysis of signatures of selective sweeps in natural populations of the house mouse. Mol Biol Evol. 2006;23(4):790-7. https://doi.org/10.1093/molbev/msj096. PubMed PMID: WOS:000235922900009.

29. Teschke M, Mukabayire O, Wiehe T, Tautz D. Identification of Selective Sweeps in Closely Related Populations of the House Mouse Based on Microsatellite Scans. Genetics. 2008;180(3):1537-45. https://doi.org/10.1534/ genetics.108.090811. PubMed PMID: WOS:000261036200023.

30. Linnenbrink M, Wang J, Hardouin EA, Kunzel S, Metzler D, Baines JF. The role of biogeography in shaping diversity of the intestinal microbiota in house mice. Mol Ecol. 2013;22(7):1904-16. https://doi.org/10.1111/mec. 12206. PubMed PMID: WOS:000316575800011.

31. Staubach F, Lorenc A, Messer PW, Tang K, Petrov DA, Tautz D. Genome Patterns of Selection and Introgression of Haplotypes in Natural Populations of the House Mouse (Mus musculus). Plos Genet. 2012;8(8). https://doi.org/10. 1371/journal.pgen.1002891. PubMed PMID: WOS:000308529300048.

32. Hasenkamp N, Solomon T, Tautz D. Selective sweeps versus introgression population genetic dynamics of the murine leukemia virus receptor Xpr1 in wild populations of the house mouse (Mus musculus). BMC Evol Biol. 2015; 15. https://doi.org/10.1186/s12862-015-0528-5. PubMed PMID: WOS: 000364373600002

33. Janousek V, Munclinger P, Wang LY, Teeter KC, Tucker PK. Functional Organization of the Genome May Shape the Species Boundary in the House Mouse. Mol Biol Evol. 2015;32(5):1208-20. https://doi.org/10.1093/molbev/ msv01 1. PubMed PMID: WOS:000355318400008.

34. Waterston RH, Lindblad-Toh K, Birney E, Rogers J, Abril JF, Agarwal P, et al. Initial sequencing and comparative analysis of the mouse genome. Nature. 2002;420(6915):520-62. https://doi.org/10.1038/nature01262. PubMed PMID: WOS:000179611600053.

35. Babik W, Taberlet P, Ejsmond MJ, Radwan J. New generation sequencers as a tool for genotyping of highly polymorphic multilocus MHC system. Mol Ecol Resources. 2009;9(3):713-9. https://doi.org/10.1111/j.1755-0998.2009. 02622.x. PubMed PMID: WOS:000265897200005.

36. Sommer S, Courtiol A, Mazzoni CJ. MHC genotyping of non-model organisms using next-generation sequencing: a new methodology to deal with artefacts and allelic dropout. BMC Genomics. 2013;14. https://doi.org/ 10.1186/1471-2164-14-542. PubMed PMID: WOS:000323064900001.

37. Kanagawa T. Bias and artifacts in multitemplate polymerase chain reactions (PCR). J Biosci Bioeng. 2003;96(4):317-23. https://doi.org/10.1016/s13891723(03)90130-7. PubMed PMID: WOS:000187530500001.

38. Lenz TL, Becker S. Simple approach to reduce PCR artefact formation leads to reliable genotyping of $\mathrm{MHC}$ and other highly polymorphic loci Implications for evolutionary analysis. Gene. 2008;427(1-2):117-423. https:// doi.org/10.1016/j.gene.2008.09.013. PubMed PMID: WOS:000261353800016.

39. Chenna R, Sugawara H, Koike T, Lopez R, Gibson TJ, Higgins DG, et al. Multiple sequence alignment with the Clustal series of programs. Nucleic Acids Res. 2003;31(13):3497-500. https://doi.org/10.1093/nar/gkg500. PubMed PMID: WOS:000183832900044

40. Bos DH, Turner SM, Dewoody JA. Haplotype inference from diploid sequence data: evaluating performance using non-neutral MHC sequences. Hereditas. 2007;144(6):228-34. https://doi.org/10.1111/j.2007.0018-0661. 01994.x. PubMed PMID: WOS:000252444100001.

41. Tamura K, Stecher G, Peterson D, Filipski A, Kumar S. MEGA6: Molecular Evolutionary Genetics Analysis Version 6.0. Mol Biol Evol. 2013;30(12):2725-9. https://doi.org/10.1093/molbev/mst197. PubMed PMID: WOS:000327793000019.

42. Stephens M, Smith NJ, Donnelly P. A new statistical method for haplotype reconstruction from population data. Am J Hum Genet. 2001;68(4):978-89. https://doi.org/10.1086/319501. PubMed PMID: WOS:000167666000019.

43. Stephens M, Donnelly P. A comparison of Bayesian methods for haplotype reconstruction from population genotype data. Am J Hum Genet. 2003; 73(5):1162-9. https://doi.org/10.1086/379378. PubMed PMID: WOS: 000186493400017 
44. Biedrzycka A, Sebastian A, Migalska M, Westerdahl H, Radwan J. Testing genotyping strategies for ultra-deep sequencing of a co-amplifying gene family: MHC class I in a passerine bird. Mol Ecol Resources. 2017;17(4):642-55. https:// doi.org/10.1111/1755-0998.12612. PubMed PMID: WOS:000403258900007.

45. Thomas M, Moeller F, Wiehe T, Tautz D. A pooling approach to detect signatures of selective sweeps in genome scans using microsatellites. Mol Ecol Notes. 2007;7(3):400-3. https://doi.org/10.1111/j.1471-8286.2007.01697.x. PubMed PMID: WOS:000246244300007.

46. Dieringer D, Schlotterer C. MICROSATELLITE ANALYSER (MSA): a platform independent analysis tool for large microsatellite data sets. Mol Ecol Notes. 2003;3(1):167-9. https://doi.org/10.1046/j.1471-8286.2003.00351.x. PubMed PMID: WOS:000181651400055.

47. Meirmans PG, Van Tienderen PH. GENOTYPE and GENODIVE: two programs for the analysis of genetic diversity of asexual organisms. Molecular Ecology Notes. 2004;4(4):792-794. https://doi.org/10.1111/j.1471-8286.2004.00770.x. PubMed PMID: WOS:000225496600081.

48. Wang HH, Church GM. Multiplexed genome engineering and genotyping methods: applications for synthetic biology and metabolic engineering. Synthetic Biology, Pt B: Computer Aided Design and DNA Assembly. 2011; 498:409-426. https://doi.org/10.1016/b978-0-12-385120-8.00018-8. PubMed PMID: WOS:000292007500018.

49. Pritchard JK, Stephens M, Donnelly P. Inference of population structure using multilocus genotype data. Genetics. 2000;155(2):945-959. PubMed PMID: WOS:000087475100039.

50. Earl DA, Vonholdt BM. STRUCTURE HARVESTER: a website and program for visualizing STRUCTURE output and implementing the Evanno method. Conservation Genetics Resources. 2012;4(2):359-361. https://doi.org/10.1007/ s12686-011-9548-7. PubMed PMID: WOS:000303536400036.

51. Evanno G, Regnaut S, Goudet J. Detecting the number of clusters of individuals using the software STRUCTURE: a simulation study. Molecular Ecology. 2005;14(8):2611-2620. https://doi.org/10.1111/j.1365-294X.2005. 02553.x. PubMed PMID: WOS:000229961500029.

52. Excoffier $L$, Lischer HEL. Arlequin suite ver 3.5: a new series of programs to perform population genetics analyses under Linux and Windows. Molecular Ecology Resources. 2010;10(3):564-567. https://doi.org/10.1111/j.1755-0998. 2010.02847.x. PubMed PMID: WOS:000276407300020.

53. Librado P, Rozas J. DnaSP v5: a software for comprehensive analysis of DNA polymorphism data. Bioinformatics. 2009;25(11):1451-1452. https://doi.org/ 10.1093/bioinformatics/btp187. PubMed PMID: WOS:000266109500026.

54. Delport W, Poon AFY, Frost SDW, Pond SLK. Datamonkey 2010: a suite of phylogenetic analysis tools for evolutionary biology. Bioinformatics. 2010; 26(19):2455-2457. https://doi.org/10.1093/bioinformatics/btq429. PubMed PMID: WOS:000282170000014.

55. Sawyer S. Statistical tests for detecting gene CONVERSION. Molecular Biology and Evolution. 1989;6(5):526-538. PubMed PMID: WOS:A1989AL48700008.

56. Prager EM, Sage RD, Gyllensten U, Thomas WK, Hubner R, Jones CS, et al. Mitochondrial-dna sequence diversity and the colonization of scandinavia by house mice from east holsten. Biological Journal of the Linnean Society. 1993;50(2):85-122. PubMed PMID: WOS:A1993MQ67300001.

57. Pezer Z, Harr B, Teschke M, Babiker H, Tautz D. Divergence patterns of genic copy number variation in natural populations of the house mouse (Mus musculus domesticus) reveal three conserved genes with major populationspecific expansions. Genome Research. 2015;25(8):1114-1124. https://doi. org/10.1101/gr.187187.114. PubMed PMID: WOS:000358957500004.

58. Abi-Rached L, Jobin MJ, Kulkarni S, McWhinnie A, Dalva K, Gragert L, et al. The Shaping of Modern Human Immune Systems by Multiregional Admixture with Archaic Humans. Science. 2011;334(6052):89-94. https://doi. org/10.1126/science.1209202. PubMed PMID: WOS:000295580300046.

59. Nadachowska-Brzyska K, Zielinski P, Radwan J, Babik W. Interspecific hybridization increases MHC class II diversity in two sister species of newts. Molecular Ecology. 2012;21(4):887-906. https://doi.org/10.1111/j.1365-294X. 2011.05347.x. PubMed PMID: WOS:000299416900009.

60. Wegner KM, Eizaguirre C. New(t)s and views from hybridizing MHC genes: introgression rather than trans-species polymorphism may shape allelic repertoires. Molecular Ecology. 2012;21(4):779-781. https://doi.org/10.1111/j. 1365-294X.2011.05401.x. PubMed PMID: WOS:000299416900001.

61. Grossen C, Keller L, Biebach I, Croll D, Int Goat Genome C. Introgression from Domestic Goat Generated Variation at the Major Histocompatibility Complex of Alpine Ibex. Plos Genetics. 2014;10(6). https://doi.org/10.1371/ journal.pgen.1004438. PubMed PMID: WOS:000338847700052.
62. Widera G, Flavell RA. The nucleotide-sequence of the murine i-e-beta-b immune-response gene - evidence for gene conversion events in class-ii genes of the major histocompatibility complex. Embo Journal. 1984;3(6): 1221-1225. PubMed PMID: WOS:A1984SU71000002.

63. Gyllensten UB, Sundvall M, Erlich HA. Allelic diversity is generated by intraexon sequence exchange at the drb1 locus of primates. Proceedings of the National Academy of Sciences of the United States of America. 1991; 88(9):3686-3690. https://doi.org/10.1073/pnas.88.9.3686. PubMed PMID: WOS:A1991FK18400037.

64. Hogstrand K, Bohme J. A determination of the frequency of gene conversion in unmanipulated mouse sperm. Proceedings of the National Academy of Sciences of the United States of America. 1994;91(21):9921-9925. https://doi. org/10.1073/pnas.91.21.9921. PubMed PMID: WOS:A1994PM13800053.

65. Zangenberg G, Huang MM, Arnheim N, Erlich H. New hla-dpb1 alleles generated by interallelic gene conversion detected by analysis of sperm. Nature Genetics. 1995;10(4):407-414. https://doi.org/10.1038/ng0895-407. PubMed PMID: WOS:A1995RM37500013.

66. Bergstrom TF, Josefsson A, Erlich HA, Gyllensten U. Recent origin of HLADRB1 alleles and implications for human evolution. Nature Genetics. 1998; 18(3):237-242. https://doi.org/10.1038/ng0398-237. PubMed PMID: WOS: 000072325000021.

67. Richman AD, Herrera LG, Nash D, Schierup MH. Relative roles of mutation and recombination in generating allelic polymorphism at an MHC class $\|$ locus in Peromyseus maniculatus. Genetical Research. 2003;82(2):89-99. https://doi.org/10.1017/s0016672303006347. PubMed PMID: WOS: 000187284500002

68. Reusch TBH, Langefors A. Inter- and intralocus recombination drive MHC class IIB gene diversification in a teleost, the three-spined stickleback Gasterosteus aculeatus. Journal of Molecular Evolution. 2005;61(4):531-U45. https://doi.org/10.1007/s00239-004-0340-0. PubMed PMID: WOS: 000232403400012

69. Spurgin LG, van Oosterhout C, Illera JC, Bridgett S, Gharbi K, Emerson BC, et al. Gene conversion rapidly generates major histocompatibility complex diversity in recently founded bird populations. Molecular Ecology. 2011; 20(24):5213-5225. https://doi.org/10.1111/j.1365-294X.2011.05367.x. PubMed PMID: WOS:000298089300010.

70. Mack SJ, Cano P, Hollenbach JA, He J, Hurley CK, Middleton D, et al. Common and well-documented HLA alleles: 2012 update to the CWD catalogue. Tissue Antigens. 2013;81(4):194-203. https://doi.org/10.1111/tan. 12093. PubMed PMID: WOS:000316628100002.

\section{Ready to submit your research? Choose BMC and benefit from:}

- fast, convenient online submission

- thorough peer review by experienced researchers in your field

- rapid publication on acceptance

- support for research data, including large and complex data types

- gold Open Access which fosters wider collaboration and increased citations

- maximum visibility for your research: over $100 \mathrm{M}$ website views per year

At BMC, research is always in progress.

Learn more biomedcentral.com/submissions 